\title{
Non-convulsive status epilepticus: a profile of patients diagnosed within a tertiary referral centre
}

\author{
S Haffey, A McKernan, K Pang
}

J Neurol Neurosurg Psychiatry 2004;75:1043-1044. doi: 10.1136/jnnp.2003.019612

Non-convulsive status epilepticus (NCSE) is an underrecognised but treatable condition with considerable associated morbidity. The files of adult admissions were reviewed over four years, during which time 50 episodes of NCSE were diagnosed in 45 patients. These findings suggest a broad age range with various states of mental acuity and an association with both learning disabilities and acute systemic infection. Approximately half of the patients had a previous diagnosis of epilepsy at the time of admission, and half had a history of generalised tonic-clonic seizures immediately before the onset of their illness.

$\mathrm{N}$ on-convulsive status epilepticus (NCSE) is a pleomorphic condition, the clinical features of which vary widely depending upon the region of the brain affected by the seizure.

NCSE was first described by Charcot in 1888 in a somnambulant patient whose condition responded to triple bromides and in 1945 Lennox demonstrated the electroclinical correlation in a patient with "petit mal status". ${ }^{1}$

At first, the condition was considered rare, ${ }^{2}$ but more recently authors have described a complex condition with a variable clinical presentation, an often under-recognised but treatable entity, associated with serious neurological and systemic morbidity and mortality.

\begin{abstract}
AIMS
Our aims were to provide a profile of patients diagnosed with NCSE to highlight any common factors in their past medical history or clinical presentation and to increase the awareness of clinicians regarding this complex and often underrecognised condition.
\end{abstract}

\section{METHODS}

We reviewed the request forms, electroencephalogram (EEG) records, radiology files, patient notes, and discharge summaries for all patients over the age of 15 diagnosed with NCSE at a large tertiary referral centre in Belfast between 1998 and 2001 .

In total, 45 adult patients were diagnosed with 50 episodes of NCSE from a total of 1829 inpatient EEGs performed during this period. Because of the pleomorphic nature of the condition, we did not attempt to subdivide the attacks into absence or complex partial status epilepticus, as suggested by Kaplan. ${ }^{3}$ He argued that the "condition may defy clear categorisation into partial or generalised types". The difficulties in interpreting EEGs in this context were reiterated by Husain when he reviewed the clinical features of patients with NCSE in 2003. ${ }^{4}$

We defined NCSE as an epileptic state lasting for more than 30 minutes, ${ }^{5}$ with two principal components:
(1) some clinically evident alteration in mental status or behaviour from baseline and

(2) seizure activity on the EEG.

A clinical and EEG effect upon administration of intravenous antiepileptic drugs was seen in 21 , cases but this was not required to fulfil the diagnostic criteria in our study.

We reviewed the request forms and patient records to establish whether the diagnosis had been suspected by the referring physician, whether there had been a previous history of epilepsy, and whether or not the episode had been preceded by a witnessed generalised tonic-clonic seizure.

The patient's mental state around the time of the EEG was also recorded, and we established whether or not intravenous antiepileptic medication had been administered during the recording of the EEG, along with the clinical and electrographic effects of such drugs.

The radiological findings were examined and the presence of comorbid conditions before the diagnosis was documented.

\section{RESULTS}

In total, 45 adult patients aged between 17 and 91 were identified. Four of the 21 female patients and one male patient had two episodes of NCSE.

Twenty five patients had a previous diagnosis of epilepsy. Of these 25 patients with a history of epilepsy, nine were felt to be suffering from NCSE at the time of an acute systemic infection. ${ }^{6}$

Twenty patients had a witnessed generalised tonic-clonic seizure preceding the diagnosis, compared with 25 in whom no history of such an event was described.

Of the 45 initial presentations, NCSE was suspected by the referring clinician in 36 cases. In a further 111 cases, the EEG repudiated a clinical suspicion of NCSE. In the nine patients in whom the diagnosis was not suspected, six had no previous history of epilepsy.

Interestingly, 28 had remote risk factors for seizures, such as previous stroke, tumour, neurosurgery, dementia, multiple sclerosis, and encephalitis or meningitis. ${ }^{7}$ Eleven of the 45 patients had a history of learning disabilities or some form of developmental delay.

We divided the group into mental state categories as determined at the time of the EEG recording (fig 1). Six $(12 \%)$ of the patients were determined to be alert and cooperative, being able to respond to simple commands and able to comply with the EEG procedure. Of these six patients the diagnosis was suspected in only two.

A further seven (14\%) patients appeared to be aware of their surroundings but not necessarily cooperative during the procedure and were unable to respond fully to simple commands.

Abbreviations: EEG, electroencephalogram; NCSE, non-convulsive status epilepticus 


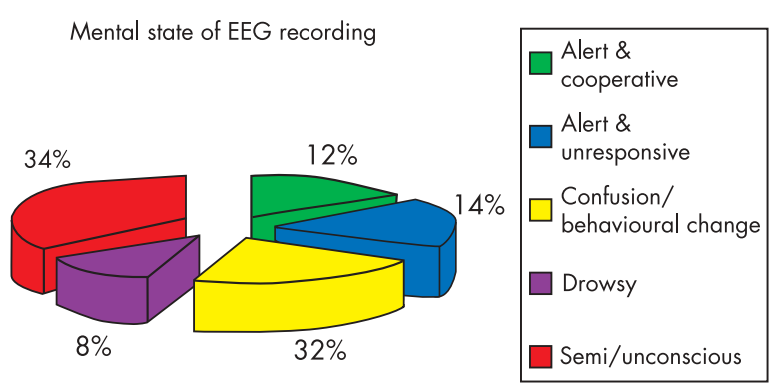

Figure 1 Mental state categories of the 45 patients at the time of electroencephalogram recording.

In almost one third of presentations, the subject was felt to be confused. Four $(8 \%)$ patients were drowsy but easily alerted with auditory stimuli. A further one third of patients were deemed to be semiconscious or unconscious at the time of the recording.

Twenty three of the 45 initial presentations were given intravenous anticonvulsants during the EEG recording. Of this group, 21 had a clinical or electroencephalographic change. On two occasions the drug appeared to be ineffective.

Twenty one patients were found to have normal cerebral imaging, as assessed by computerised tomography or magnetic resonance imaging, whereas six demonstrated changes in the temporal lobes consistent with previous or recent herpes simplex encephalitis.

\section{DISCUSSION}

NCSE can present at any age and the clinical features may vary widely. The diagnosis was more likely to be suspected in patients with a previous history of epilepsy; however, 20 of the 45 patients in our series had no previous history of epilepsy. The condition was also less likely to be suspected in patients who appeared to be alert and relatively cooperative.

The diagnosis should be considered as a differential diagnosis in patients in a prolonged post-ictal state because 20 patients were known to have had a generalised tonicclonic seizure preceding the diagnosis; however, the absence of a clear history of seizure activity should not diminish the clinician's suspicions of NCSE as a possible diagnosis in patients with an altered mental state.

As highlighted by Kaplan in an abstract at the American Clinical Neurophysiology Society, systemic infection may be a contributory factor in the development of NCSE, especially in known epileptic patients. ${ }^{78}$ Conversely, a febrile patient or a patient with confirmed infection should not be assumed to be confused simply on the basis of a concurrent illness. NCSE should be suspected particularly in confused elderly patients with a change in mental state or behaviour.

\section{LIMITATIONS}

We acknowledge the limitations of a study of this nature. The viewpoint is retrospective and not population based, but rather a review of hospital records within a large tertiary referral centre. We collected data on adults only, although status epilepticus is thought to be more common in children. ${ }^{6}$ We did not concentrate on complications, because it is difficult to determine which are directly attributable to NCSE and which result from the underlying aetiology. The study focused rather on the risk factors to heighten awareness of the condition among physicians.

\section{Authors' affiliations \\ S Haffey, A McKernan, K Pang, Department of Clinical \\ Neurophysiology, Royal Victoria Hospital, Belfast BT12 6BA, UK \\ Competing interests: none declared}

Correspondence to: Dr S Haffey, Department of Clinical Neurophysiology, Royal Victoria Hospital, Belfast BT12 6BA, UK; haffwit@hotmail.com

Received 27 May 2003

In revised form 18 September 2003

Accepted 1 November 2003

\section{REFERENCES}

1 Lennox W. The petit mal epilepsies: their treatment with tridione. JAMA 1945; 129:1069-73.

2 Krumholz Allan. Epidemiology and evidence for morbidity of nonconvulsive status epilepticus. J Clin Neurophysiol 1999;16:314-22.

3 Kaplan PW. Non-convulsive status epilepticus: to lump or to split?" Epilepsia 1994;35(suppl 8):9.

4 Husain AM. Non-convulsive status epilepticus: usefulness of clinical features in selecting patients for urgent EEG. J Neurol Neurosurg Psychiatry 2003;74:189-91.

5 Kaplan PW. Assessing the outcomes in patients with non-convulsive status epilepticus: non-convulsive status epilepticus is undiagnosed, potentially overrated and confounded by co-morbidity. J Clin Neurophysiol 1999; 16:341-52.

6 Dunne JW. Non-convulsive status epilepticus: a prospective study in an adult general hospital. Quart J Med 1987;62:117-26.

7 Kaplan PW. Triggers of NCSE in the elderly. J Clin Neurophysiol 2003;20:207-25.

8 Fountain NB. Status epilepticus: risk factors and complications. Epilepsia 2000;41(suppl 2):523-30. 